UCRL-JC-128281

PREPRINT

\title{
Directions of ICF Research in the United States
}

\author{
W. J. Hogan
}

E. M. Campbell

This paper was prepared for submittal to Thirteenth International Conference on Laser Interaction and Related Plasma Phenomena

Monterey, CA

April 13-18, 1997

August 1, 1997

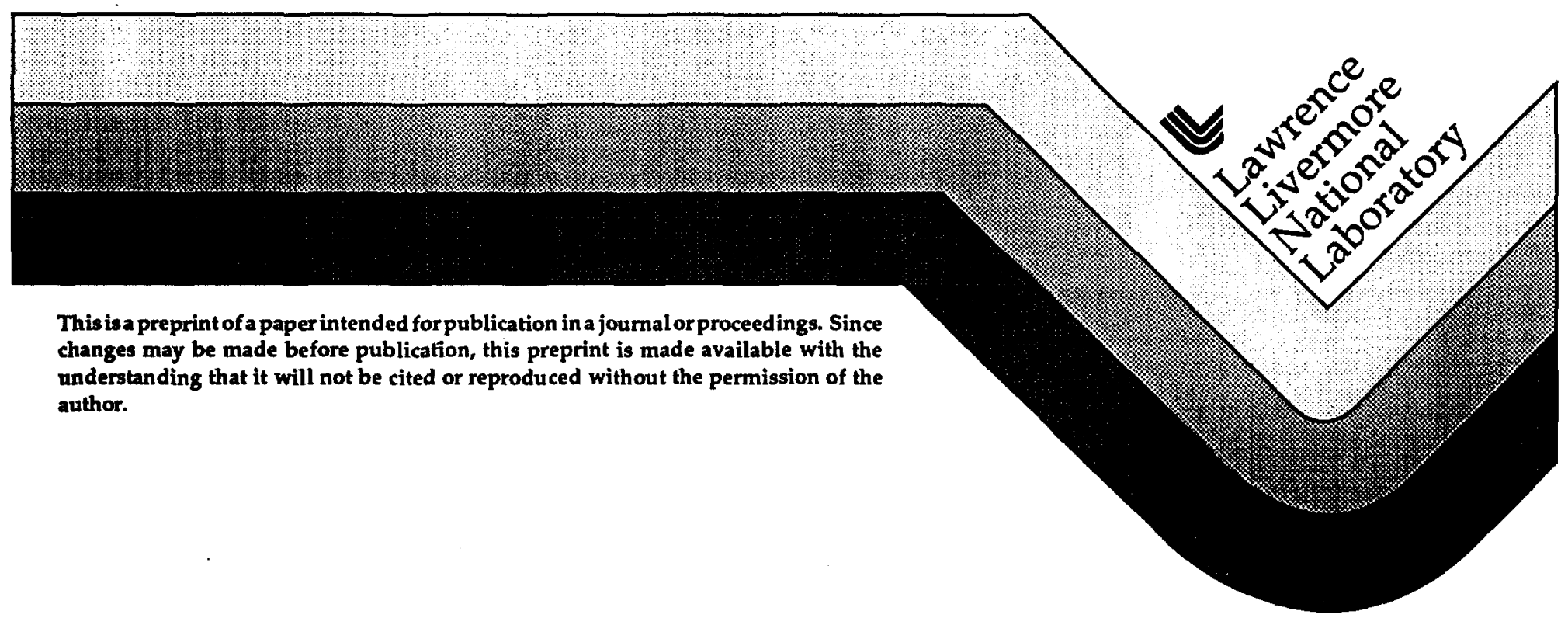




\section{DISCLAMER}

This document was prepared as an account of work sponsored by an agency of the United States Government. Neither the United States Government nor the University of California nor any of their employees, makes any warranty, express or implied, or assumes any legal liability or responsibility for the accuracy, completeness, or usefulness of any information, apparatus, product, or process disclosed, or represents that its use would not infringe privately owned rights. Reference herein to any specific commercial product, process, or service by trade name, trademark, manufacturer, or otherwise, does not necessarily constitute or imply its endorsement, recommendation, or favoring by the United States Government or the University of California. The views and opinions of authors expressed herein do not necessarily state or reflect those of the United States Government or the University of California, and shall not be used for advertising or product endorsement purposes. 


\title{
Directions of ICF Research in the United States
}

\author{
William J. Hogan and E. Michael Campbell
}

\author{
Lawrence Livermore National Laboratory \\ 7000 East Avenue, Livermore, Califormia 94550
}

\section{INTRODUCTION}

Inertial Confinement Fusion (ICF) research in the United States is in a dramatic upswing. Technical progress continues at a rapid pace and with the start of construction of the National Ignition Facility (NIF) this year the total U.S. budget for ICF for fiscal year 1997 stands at $\$ 380$ million. The NIF is being built as an essential component of the U.S. Stockpile Stewardship and Management Program, which has been formulated to assure the continued safety, reliability and performance of the downsized nuclear weapons stockpile in the absence of nuclear tests. Thus the increase in funding originates in the Congressional armed services committees and is managed by Defense Programs of the Department of Energy. The NIF, however, is a fundamental research tool that will be of great benefit beyond its mission within the nuclear weapons program. Its experiments will promote fusion energy development and will open new areas of basic scientific research. This paper will discuss some of the directions that ICF research is now taking, the progress on the NIF Project, and the potential impact that these developments are likely to have on fusion energy development and on certain areas of the basic sciences.

To be successful in today's budget-conscious world, which is skeptical of big science, any scientific program requiring large facilities must have several attributes. Among these are clear and widely accepted goals with near term measures of progress and a flexible and affordable path to achieving those goals in a reasonable length of time. It is also highly desirable if the program has one or more defensible secondary goals and some near term spin-off benefits. The ICF program has these attributes and that may account for its success during times when many large scientific programs are having difficulties.

The goal of preserving the safety and reliability of existing nuclear weapons while not developing new types of weapons and not performing nuclear tests is one that receives general support from most of the political spectrum. While extreme positions on both sides of the nuclear weapons issue have been taken, the great majority of people take a more measured approach to nuclear arms control. That ICF, through the NIF can make an essential contribution to this moderate position is the basis of much of our political strength today. It is a great bonus that 
achieving ignition on the NIF will also play such a key role in the development of inertial fusion as a source of electric power production, that it will contribute so much to the basic sciences and that it will have a major impact on advancing technologies that will increase industrial capabilities.

\section{THE NIF PROJECT}

The NIF Project has made significant progress in the last two years. The preliminary engineering design phase has been completed and the cost of the new baseline design (Figure 1) has been re-estimated. At $\$ 1.2$ billion, the cost of NIF has risen less than $10 \%$ compared to the cost estimate for the conceptual design published in 1994. While the basic requirements for NIF remain the same (e.g. 192 beamlines, $1.8 \mathrm{MJ}, 500 \mathrm{TW}$ of $0.35 \mu \mathrm{m}$ light), several new options have been added. The NIF design illustrates many of the features of IFE development and will play an essential role in addressing IFE feasibility. For example, the NIF will now be capable of both direct and indirect drive experiments (Figure 2). Another new capability added is the ability to begin experiments two years before the end of NIF construction. The modular design of NIF allows bringing one bundle of

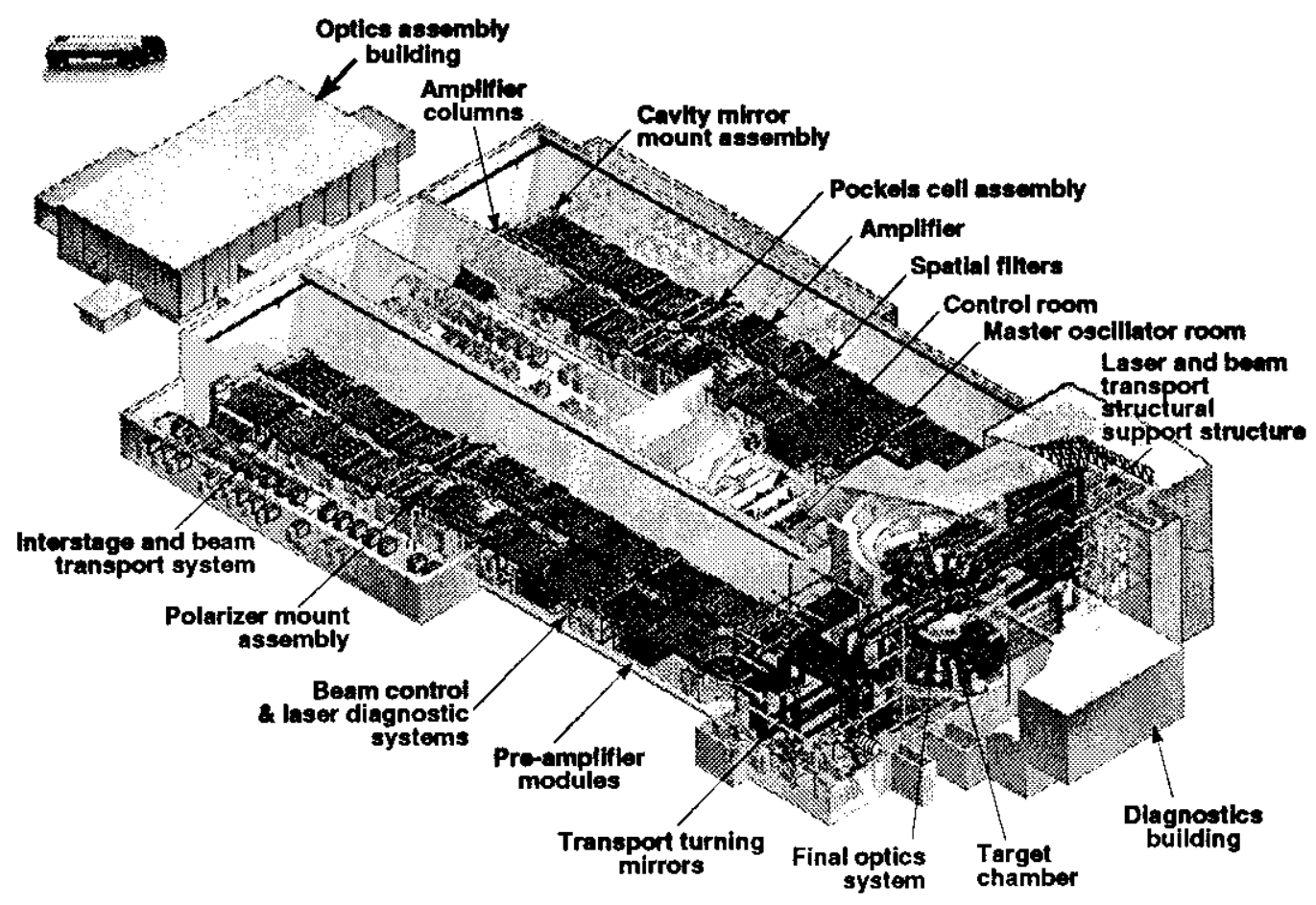

40-00-0996-2100Ypb01

FIGURE 1. The final design of the National Ignition Facility at LLNL includes the laser and target area building and an optical assembly building containing clean rooms for assembly and maintenance functions. 
eight beamlines up for experiments in 2001 while construction proceeds in the other laser bay. With more than $75 \mathrm{~kJ}$ of energy, one bundle will be equivalent to two Nova lasers. The entire facility will be complete in 2003 and at full energy in 2004.

\section{INERTIAL FUSION ENERGY RESEARCH}

For the energy mission, target ignition and modest energy gain will represent the scientific proof-of-principle of inertial fusion as an energy source. The critical issue for inertial fusion energy (IFE) is whether ignition can be accomplished at a sufficiently small driver size (i.e. cost) that inertial fusion energy is economically reasonable. When ignition is achieved on NIF, we will then map out an entire set of ignition and gain curves and determine the minimum energy at which ignition and gain can be achieved. Figure 3 shows several sets of ICF gain curves for direct and indirect targets for a variety of assumptions about hohlraum drive temperature, target surface finish and other variables. Also shown are the estimated gains for the fast ignitor concept to be discussed below. NIF
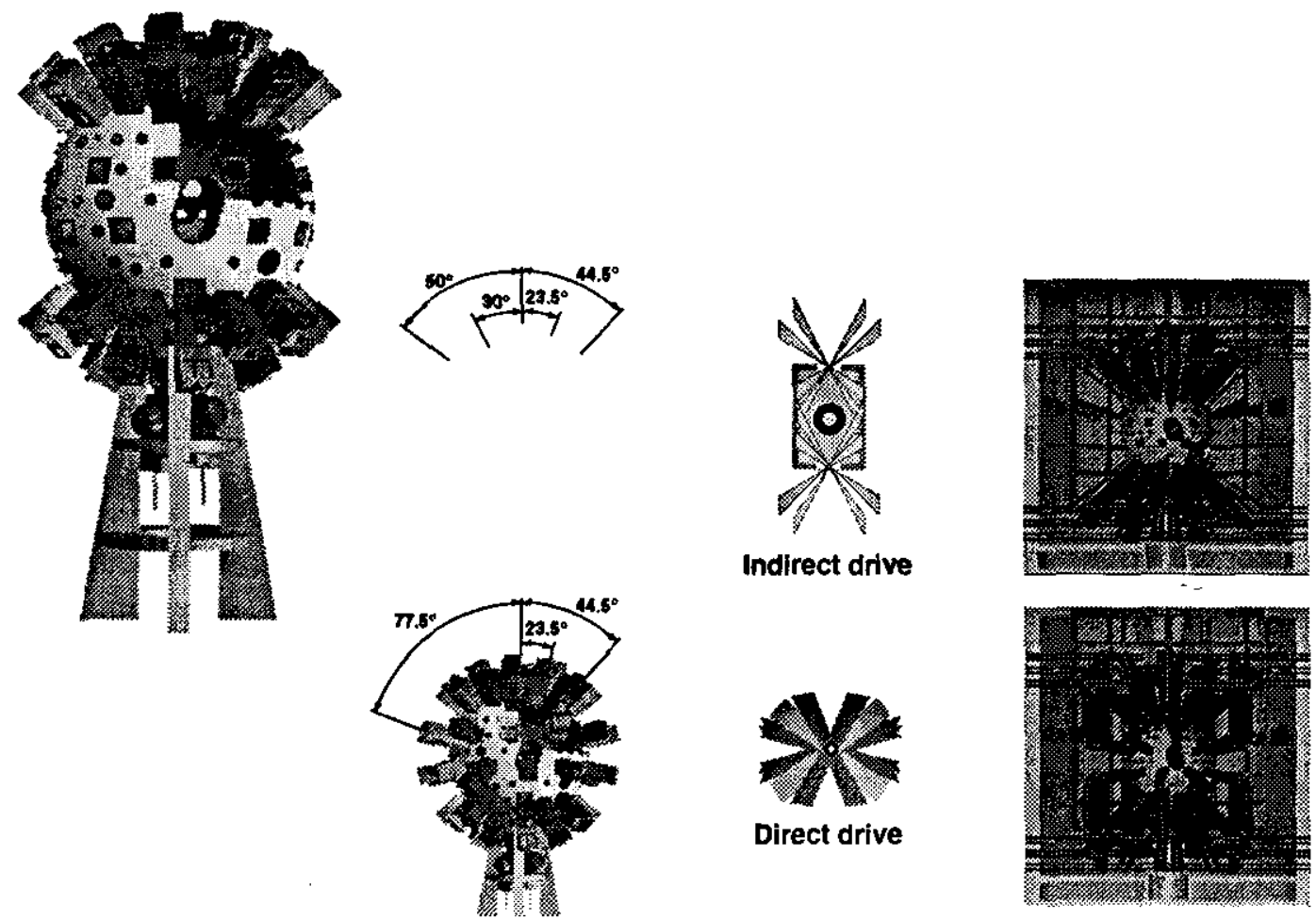

Indirect drive

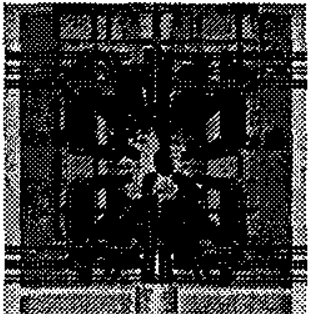

10.00-1096-2482B

FIGURE 2. The NIF will be able to support both indirect and direct drive target experiments. The target area can be reconfigured for direct drive targets by moving 24 of the $482 \times 2$ beam clusters from the polar to the equatorial region. 
experiments will examine target performance as a function of these variables to determine how an engineering test facility and, subsequently, a power plant must be designed. For example, determining target performance as a function of target surface finish will determine the precision with which targets must be mass manufactured. NIF will be capable of doing experiments relevant to all proposed target types, regardless of driver type. For example, by altering the laser illumination geometry, NIF experiments will be able to examine X-ray transport and capsule performance issues for targets designed for heavy ion drivers. The only heavy ion target issue that NIF cannot address, of course, is the deposition of energy in the target by the ions themselves. NIF ignition experiments will gather data necessary to design an engineering test facility by examining the reaction of various possible first walls and internal blankets to the effects of a target explosion.

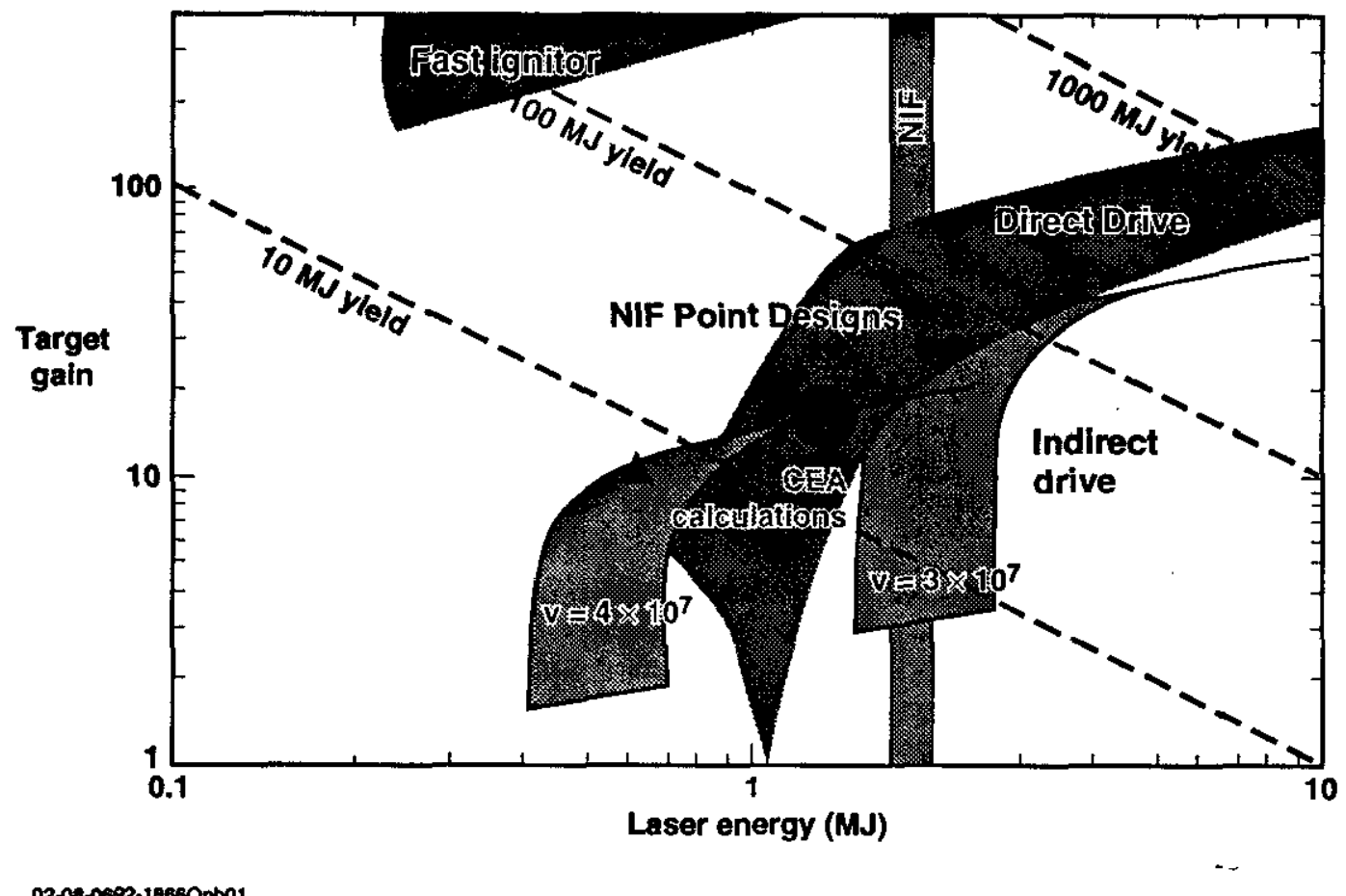

FIGURE 3. NIF will map out the ignition and gain curves for several target concepts. Shown are two sets of gain curves for indirect drive targets using different hohlraum drive temperatures (the numbers indicate resulting implosion velocities), one set for direct drive targets, the predictions for indirect drive from the CEA, and the estimated "fast ignitor" gains. (02-08-0692-1866Q)

Another aspect of IFE research is the question of what driver will be best for power production. At present, the heavy ion driver is the front runner in the United States. Ion accelerators are reliable, have high pulse rates and can convert electricity to beam energy with efficiencies of up to $35 \%$. The critical question for heavy ion accelerators is whether they can economically accelerate a high current (kiloamps) while preserving a sufficiently good emittance (i.e. high beam brightness) that the high temperatures required for target ignition are achieved. 
Unfortunately, heavy ion accelerators are not very scaleable - that is, they cannot demonstrate their ability to achieve the necessary target intensities at small accelerator size. The Lawrence Berkeley National Laboratory and LLNL are collaborating to explore ion accelerator concepts like the recirculating induction accelerator, that may be able to provide the necessary target conditions reasonably economically.

We are also exploring the future of solid state lasers. To be a good power plant driver, solid state lasers must demonstrate high efficiency in converting electricity into beam energy and high pulse repetition rate. The diode pumped solid state laser offers such promise. Pumping a solid state laser with diodes instead of flash lamps can drastically improve the efficiency of such lasers. The NIF flash-lamp-pumped Nd:glass laser will be about $1 \%$ efficient compared with Nova which has an efficiency of about $0.1 \%$. A reasonable driver must have an efficiency greater than about $10 \%$ to effectively use targets with a gain of about 100 (the product of driver efficiency and target gain must be above about 10 for an economic power plant). Laser diodes emit light in a very narrow bandwidth which can be chosen to match the absorption bandwidth of the lasing ion/host material so that the efficiency of the combination can be above $10 \%$. We have found that ion/host combinations like Yb:S-FAP, Ytterbium in a crystalline material (Strontium fluoro-apatite), can accomplish this goal and will also have many of the other necessary characteristics for a laser driver, e.g. the ability to extract heat rapidly without creating destructive thermal gradients so that high repetition rate is achievable and a sufficiently long spontaneous emission lifetime that the laser diode arrays are efficiently used (Figure 4). In other experiments we have demonstrated the ability to cool laser discs while preserving beam quality, to economically produce diode arrays with high average power, and to grow the necessary quality of $\mathrm{Yb}: \mathrm{S}-\mathrm{FAP}$ crystals. We have begun a project on internal funds to build a $100 \mathrm{~J}$ module of such a high average power laser (code named Mercury) to demonstrate an integrated, modular design.

Many issues related to these lasers remain (cost, overall efficiency, beam smoothing) and must be addressed in the future. Other technologies, such as $\mathrm{KrF}$ lasers also show promise for IFE drivers.

Another aspect of our IFE research is to explore new target concepts that offer the potential of achieving higher gain at reasonable drive energy. The fast ignitor is such a concept. Conventional ICF targets require that the DT fuel be cold and compressed to high density while a central portion (about $2 \%$ of the fuel) is heated to ignition. This requires a high degree of precision. The fast ignitor concept suggests that cold fuel be compressed to high density without worrying about a central hot spot. After compression a very short pulse laser supplies heating to a tiny fraction of the fuel at the edge of the compressed core. The pulse must be short enough that the energy required for ignition is deposited in a time short compared to the time for that energy to be transported away from the hot spot. The development of the chirped-pulse-amplification laser has made this concept feasible. Very short laser pulses are chromatically spread with gratings so that a long, low power pulse can be amplified. The amplified pulse is recompressed after amplification with high damage threshold gratings so that the resulting short pulse reaches very high power. Using one beam of Nova, we have produced a beam of $600 \mathrm{~J}$ with a pulse length of $450 \mathrm{fs}$, resulting in a power of about 1.3 PW. We are now undertaking the experiments necessary to determine how the short laser pulse penetrates the plasma produced by compressing a fuel 

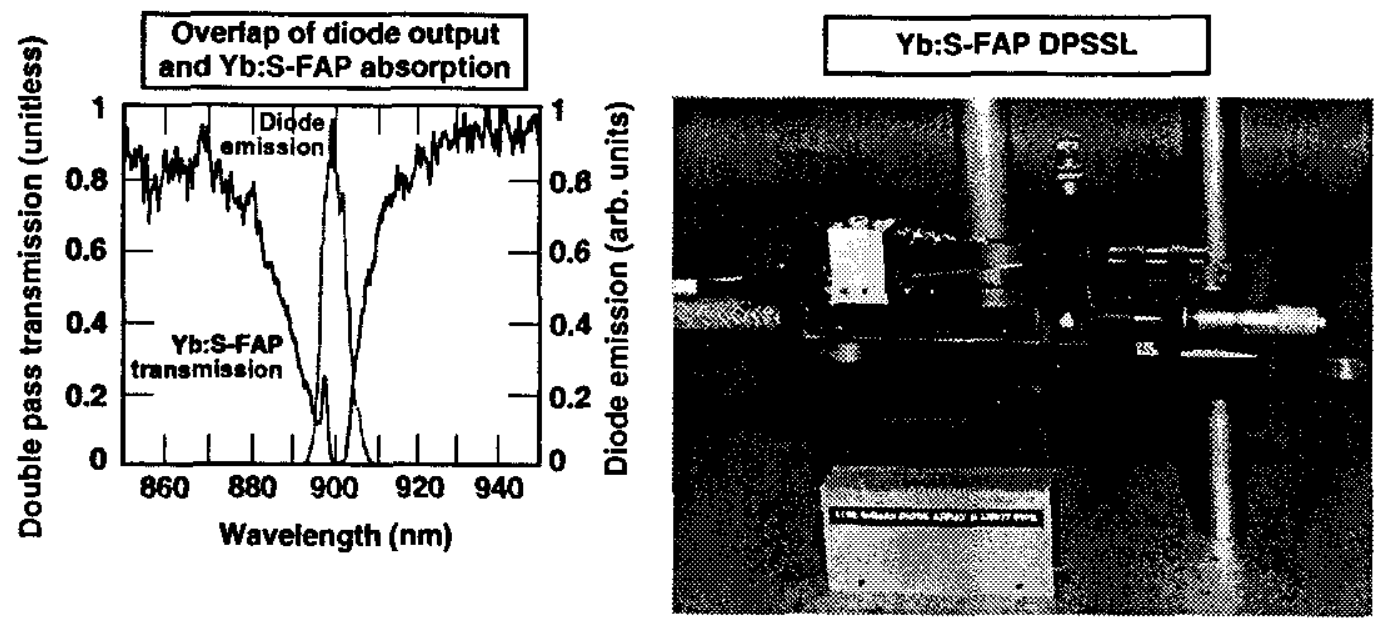

70-00-1194-3746pbo1

FIGURE 4. A small Yb:S-FAP diode pumped solid state laser (shown at right) was demonstrated in 1994. The overlap of the diode emission band and the Yb:S-FAP absorption band has resulted in an optical slope efficiency of 9.1\%. (70-00-1194-3746 but use only the top two figures, include the words above the two figures in the boxes.)

capsule and what the coupling efficiency is. If these experiments prove promising, we will add this capability to NIF to explore whether the very high gains shown in Fig. 3 for the fast ignitor can be achieved with the drive energy available on NIF. This could mean that gains sufficient for a power plant might be achievable with the NIF laser.

\section{SCIENCE ON HIGH-INTENSITY LASERS AND THE NIF}

The temperatures and pressures achievable on NIF are not only those obtained in a nuclear weapon explosion but they are the same as those that exist at the center of the sun and other stars. Thus, astrophysicists are finding that experiments using high intensity lasers can help them gain a better understanding of various astrophysical phenomena. An recent experiment on Nova produced a better measurement of the opacity of iron at temperatures and densities typical of those that exist in the interior of Cepheid variable stars. This measurement led to a better theory and calculational model of Cepheid variables and this, in turn, led to a new estimate of the age of the universe. Such are the possibilities when a new capability such as high intensity laser experiments become available. Current experiments on Nova are exploring the physics of Rayleigh-Taylor instabilities as they may exist in Supernova 1987a. Such instabilities explain "anomalies" in detected radiation from the Super Nova. Nova experiments are attempting to simulate the instability growth so that the hydrodynamic models of this phenomenon can be tested and improved, perhaps, those models can be tested and used to predict the timing and value of the expected $\mathrm{X}$-ray signal during the expected collision. 
Experiments are also underway on Nova now to explore the collision hydrodynamics of ejecta from the Super Nova with surrounding material scheduled to occur in 1999.

The NIF will greatly expand our capability to do experiments applicable to these and other basic science topics. With a laser energy and power more than $\mathbf{4 0}$ and 10 times that of Nova, these high temperature and density phenomena can be followed for much greater times and higher atomic number materials can be used in experiments that simulate relevant stellar conditions. Instability growth experiments can be followed beyond the linear growth regime. Experiments could even be done to examine the fundamental nucleosynthesis processes that occurred in the early moments of the "big bang". NIF will truly bring astrophysics into an earthbound laboratory.

\section{EXPLOITATION OF NEAR TERM SPIN-OFF BENEFITS}

Making progress on inertial fusion has required pressing the state of the art in many areas of science and technology. Not surprisingly, many of these technical advances can be beneficial for other purposes if only there is the vision to recognize the potential and a little exploratory funding to demonstrate the application. Paying some attention to these "spin-off" applications helps us demonstrate that ICF is beneficial to immediate, societal needs even as it works toward its long term goals.

At LLNL we are exploiting several of these near term applications of ICF technology. A recent study by Bay Area Economics, overseen by an Academic Advisory Panel from the University of California, Berkeley, concluded that spinoff applications are likely to dramatically impact industries representing a significant portion of the U. S. gross domestic product. Two types of technologies were examined in detail - EUV lithography and Micropower Impulse Radar (MIR).

The EUV lithography capability arose from ICF's need to diagnose the performance of targets and handle X-rays. Our development of, for example, Xray optics, laser plasma sources, and X-ray lasers gave us an experience base that allowed putting together a concept for lithography using extreme ultraviolet radiation. This development may allow fabrication of chips with a feature size much smaller than current capabilities that use optical light. Industry experts note that the process being developed by a consortium of LLNL, Sandia National Laboratories, and Lawrence Berkeley National Laboratory is absolutely unique and offers great promise. A large amount of industry funds have been committed to demonstrate a prototype of this concept over the next three years. Our federal sponsors are taking great pleasure in the fact that we have made this technology available to industry for near term exploitation.

MIR technology also arose from our need to diagnose ICF targets. The needs for picosecond time resolution led to the invention of a transient digitizer with remarkable capabilities. The engineer involved noted that if he added an antenna to this circuit, he would have a micropower radar that may have useful commercial applications. The result has exceeded our most optimistic projections. So far more than 30 licenses have been granted to industry with such applications as collision avoidance radar for automobiles, security systems, liquid level sensors, and even a low cost "stud finder" that can find a wooden stud behind 
sheet rock. Governmental applications include detecting the heartbeat of live victims under rubble from a disaster, locating buried plastic mines, and finding hidden flows in bridges so they can be repaired before a disaster occurs.

These are only two examples of the many spin-off applications that we are examining. Others will have impact on, for example, the medical use of lasers, better flat panel displays, and cheaper large precision optics. We know how to make the advanced technologies available to society now even while we continue to use them for our long term goals. This viewpoint brings home to the average citizen that an investment today in these technologies has a very big payoff indeed, both in the near term and the long term.

\section{Conclusions}

Developments in the last few years have made ICF technology more available worldwide. The December, 1993 decision to declassify all ICF ignition target information, whether direct or indirectly driven, opened the way for international cooperation. As a result, more than $80 \%$ of present Nova and future NIF experiments will be unclassified. The recent studies of the impact operation of NIF will have on nonproliferation have established that NIF can have a positive influence on international nonproliferation and arms control goals. Follow-up specific recommendations on NIF policy and management processes show that these goals can be realized. This is, NIF can fulfill its U.S. stockpile stewardship mission and at the same time be completely consistent with the letter and spirit of the nonproliferation and arms control goals of the nuclear nonproliferation treaty (NPT) and the Comprehensive Test Ban Treaty. We can work together internationally to exploit the non-military applications of ICF and NIF in energy, science, and technology.

*This work was performed under the auspices of the U.S. Department of Energy by Lawrence Livermore National Laboratory under contract No. W-7405-Eng-48. 


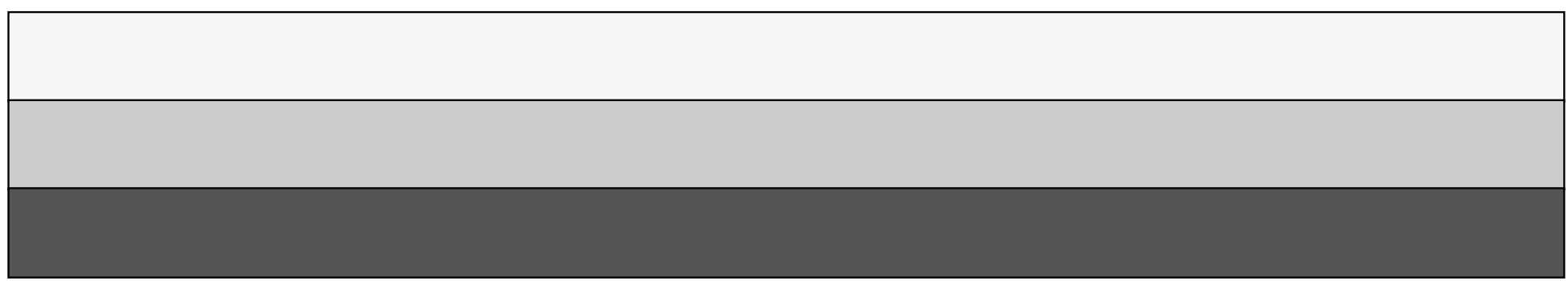

